\title{
Dermoscopy Findings of Pseudolymphomatous Folliculitis
}

\author{
Taku Fujimura Takanori Hidaka Akira Hashimoto \\ Setsuya Aiba
}

Department of Dermatology, Tohoku University Graduate School of Medicine, Sendai, Japan

\section{Key Words}

Pseudolymphomatous folliculitis · Dermoscopy · Pseudolymphoma $\cdot$ CD1a

\begin{abstract}
Pseudolymphomatous folliculitis (PLF), which clinically mimicks cutaneous lymphoma, is a rare manifestation of cutaneous pseudolymphoma and cutaneous lymphoid hyperplasia. Here, we report on a 45-year-old Japanese woman with PLF. Dermoscopy findings revealed prominent arborizing vessels with small perifollicular and follicular yellowish spots and follicular red dots. A biopsy specimen also revealed dense lymphocytes, especially CD1a+ cells, infiltrated around the hair follicles. Without any additional treatment, the patient's nodule rapidly decreased. The presented case suggests that typical dermoscopy findings could be a possible supportive tool for the diagnosis of PLF.
\end{abstract}

\section{Introduction}

Pseudolymphomatous folliculitis (PLF) was first described as a variant of pseudolymphoma. It is characterized by a dense lymphoid infiltrate accompanied by hyperplastic hair follicles [1, 2]. Clinically, PLF is sometimes difficult to differentiate from primary malignant cutaneous lymphomas that may present as solitary nodules. A diagnosis of PLF is made based on hypertrophic hair follicles, perifollicular S100+ and CD1a+ dendritic cells, and negative clonal gene rearrangement study findings [2, 3]. However, sometimes it takes a long time to achieve the final diagnosis. In this report, we present the characteristic dermoscopic features of PLF based on the typical histological features. 


\section{Case Report}

A 45-year-old Japanese woman visited our outpatient clinic with a two-month history of a developing, asymptomatic nodule on her nose. On her initial visit, physical examination revealed a red, dome-shaped nodule, $15 \times 14 \mathrm{~mm}$ in size on the right side of her nose (fig. 1a). Dermoscopy revealed prominent multiple perifollicular and follicular yellowish spots, follicular red dots, and arborizing vessels (fig. 1b). A biopsy specimen showed a dense lymphocytic infiltrate containing numerous histiocytes that surrounded and infiltrated hypertrophic hair follicles (fig. $2 a$ ). The infiltrate was separated from the epidermis by a grenz zone (fig. $2 \mathrm{~b}$ ). There was no reactive pattern in follicular centers. Infiltrated cells were medium-sized with a high nuclear/cytoplasmic ratio and prominant nucleoli (fig. 2b). Immunohistochemical staining revealed that the infiltrate consisted of a mixed population of $\mathrm{B}(\mathrm{CD} 79 \mathrm{a}+)$ and $\mathrm{T}(\mathrm{CD} 2+, \mathrm{CD} 3+, \mathrm{CD} 4+, \mathrm{CD} 5+$, and $\mathrm{CD} 7+)$ lymphocytes. The Ki67 score was approximately 20\%. CD1a+ cells were densely infiltrated around the hair follicles (fig. 2c). Based on the above data, we diagnosed PLF. One month after the skin biopsy, the nodule spontaneously decreased. Six months after the remission of the nodule, there was no sign of local recurrence.

\section{Discussion}

PLF is a rare manifestation of cutaneous pseudolymphoma and cutaneous lymphoid hyperplasia. As atypical lymphocytes can be observed in PLF, they must be differentiated from primary malignant cutaneous lymphomas that may present as solitary nodules [4-7]. However, in contrast to lymphomas, lymphocytes in PLF show no bias in their $\kappa-/ \lambda$-chain positive B cell or CD4+/CD8+ T cell ratio $[2,3]$. Moreover, the distribution of CD1a+ dendritic cells around hair follicles is a typical histological feature of PLF and not a diagnostic feature of cutaneous lymphomas [2, 3]. Though these histological findings are indispensable for the diagnosis of PLF, sometimes it takes a long time to achieve the final diagnosis. For the above reasons, another diagnostic tool for the rapid diagnosis for PLF is necessary.

Dermoscopy is a valuable, noninvasive, widely used technique which improved the diagnostic accuracy for skin cancer [8]. It allows in vivo observation of the skin with visualization of morphological structures in the epidermis and papillary dermis, which otherwise would not be discernible to the naked eye. For skin cancers, dermoscopy has had a significant impact on the early diagnosis of solid skin cancers including melanoma, basal cell carcinoma, Bowen's disease, actinic keratosis, and squamous cell carcinoma [8]. With regard to lymphoproliferative disorders, recently, Moura et al. [9] reported the dermoscopy findings of lymphomatoid papulosis to define dermoscopic criteria associated with the different stages of the disease. Interestingly, in the present case there were typical dermoscopy features, which suggest the dense infiltrate of CD1a cells around the hair follicles and prominent arborizing vessels. Torres et al. [10] also reported, in conjunction with scalp sarcoidosis, that dermoscopy findings of perifollicular and follicular yellowish spots are connected with dense infiltration of cellular components. Prominent follicular red dots, as previously described by Tosti et al. [11], were similar to those of scalp discoid lupus erythematosus. In summary, the present dermoscopy findings add another diagnostic feature for follicular disorders on the scalp and lymphoproliferative disorders. Though the present findings are limited because they came from a single case only, they might be supportive for the early diagnosis of PLF. 

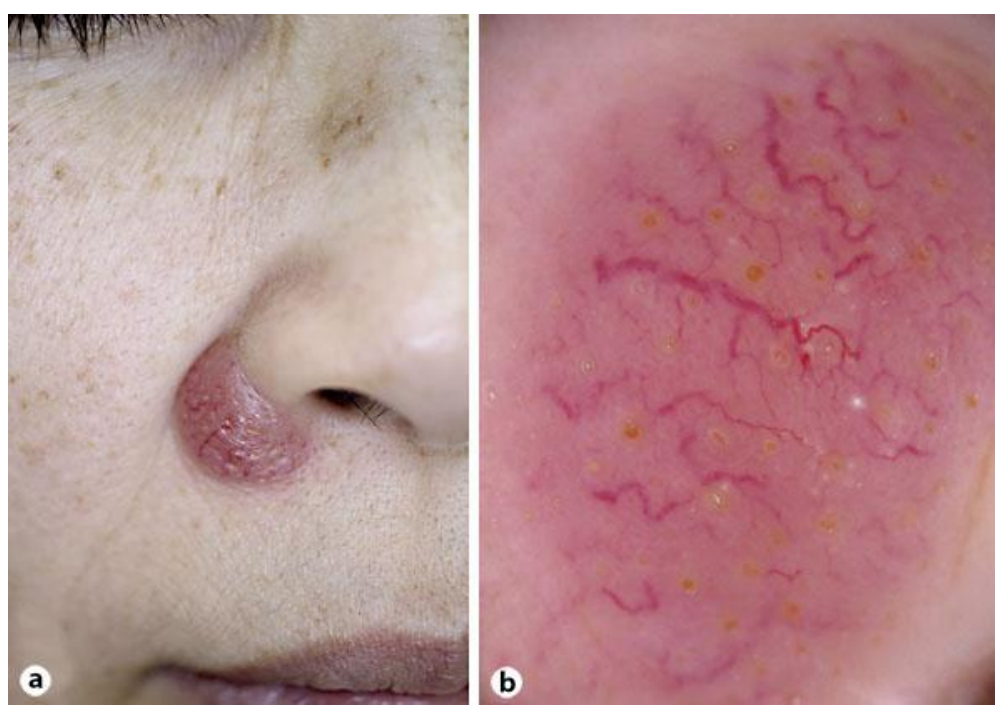

Fig. 1. A red, dome-shaped nodule, $15 \times 14 \mathrm{~mm}$ in size on the right side of the nose (a). Prominent multiple perifollicular and follicular yellowish spots, follicular red dots, and arborizing vessels (b).
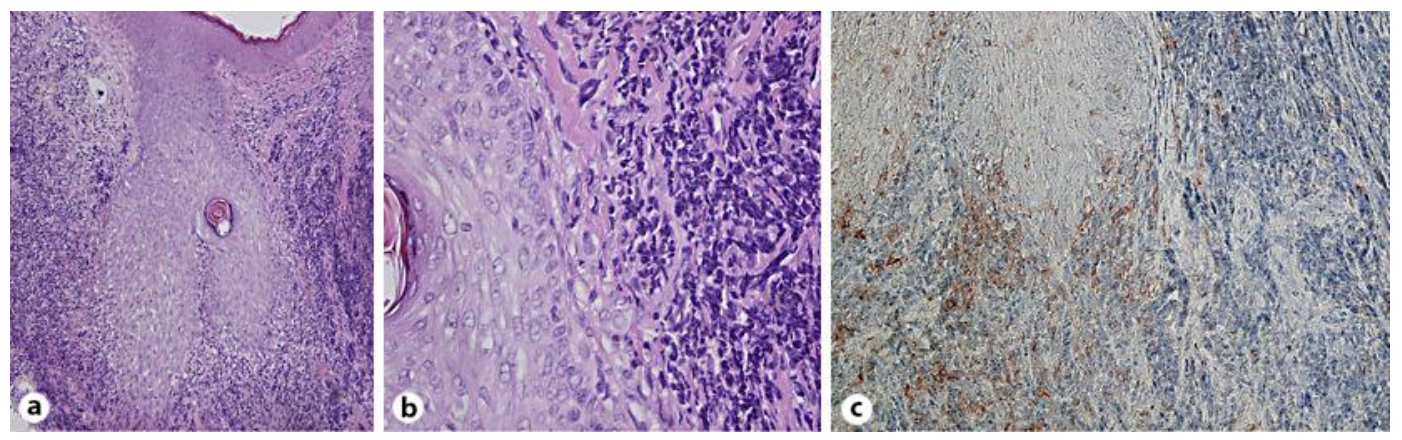

Fig. 2. A dense lymphocytic infiltrate containing numerous histiocytes that surrounded and infiltrated hypertrophic hair follicles. The infiltrate is separated from the epidermis by a grenz zone. There was no reactive pattern to the follicular centers (a). Infiltrated cells were medium-sized with a high nuclear/cytoplasmic ratio and prominant nucleoli (b). CD1a+ cells were densely infiltrated around the hair follicle (c). Original magnification, $\times 100(a), \times 400(b), \times 200(c)$. 


\section{References}

1 Mc Nutt NS: Cutaneous lymphohistiocytic infiltrates stimulating malignant lymphoma; in Murphy GF, Mihm MC (eds): Lymphoproliferative Disorders of the Skin. Boston, Butterworths, 1986, pp 256-285.

2 Kakizaki A, Fujimura T, Numata I, Hashimoto A, Aiba S: Pseudolymphomatous folliculitis on the nose. Case Rep Dermatol 2012;4:27-30.

-3 Kwon EJ, Kristjansson AK, Meyerson HJ, Fedele GM, Tung RC, Sellheyer K, Tuthill RJ, Honda KS, Gilliam AC, McNiff JM: A case of recurrent pseudolymphomatous folliculitis: a mimicking of cutaneous lymphoma. J Am Acad Dermatol 2009;60:994-1000.

-4 Arai E, Okubo H, Tsuchida T, Kitamura K, Katayama I: Pseudolymphomatous folliculitis: a clinicopathologic study of 15 cases of cutaneous pseudolymphoma with follicular invasion. Am J Surg Pathol 1999;23:1313-1319.

5 Swerdlow SH, Campo E, Harris NL, Jaffe ES, Pileri SA, Stein H, Thiele J, Vardiman JW: WHO Classification of Tumours of Haematopoietic and Lymphoid Tissues, Fourth Edition. Lyon, IARC Press, 2008, pp 145147

-6 Fujimura T, Aiba S, Yoshino Y, Kuroki S, Kimura Y, Kikuchi K, Kunii T, Matsunaga J, Matsushima K, Tagami H: CCR4 expression by atypical T cells in systemic pilotropic lymphoma: its behaivior under treatment with interferon gama, topical PUVA and systemic retinoid. Dermatology 2004;208:221-226.

-7 Van Doorn R, Scheffer E, Willemze R: Follicular mycosis fungoides, a distinct disease entitiy with or without associated follicular mucinosis: a clinicopathologic and follow-up study of 51 patients. Arch Dermatol 2002;138:191-198.

8 Fargnoli MC, Kostaki D, Piccioni A, Micantonio T, Peris K: Dermoscopy in the diagnosis and management of non-melanoma skin cancers. Eur J Dermatol 2012, E-pub ahead of print.

99 Moura FN, Thomas L, Balme B, Dalle S: Dermoscopy of lymphomatoid papulosis. Arch Dermatol 2009;145:966-967.

10 Torres F, Tosti A, Misciali C, Lorenzi S: Trichoscopy as a clue to the diagnosis of scalp sarcoidosis. Int J Dermatol 2011;50:358-361.

11 Tosti A, Torres F, Misciali C, Vincenzi C, Starace M, Miteva M, Romanelli P: Follicular red dots: a novel dermoscopic pattern observed in scalp discoid lupus erythematosus. Arch Dermatol 2009;145:14061409. 